\title{
Title: Cross-cultural effects of color, but not morphological masculinity, on perceived attractiveness of men's faces
}

Running Head: Cross-cultural color, masculinity and attractiveness

Ian D Stephen ${ }^{1}$, Isabel M L Scott ${ }^{2}$, Vinet Coetzee ${ }^{3}$, Nicholas Pound ${ }^{4}$, David I Perrett ${ }^{5}$, Ian S Penton-Voak ${ }^{6}$

${ }^{1}$ School of Psychology, University of Nottingham Malaysia Campus Department of Archaeology and Anthropology, University of Bristol, UK

${ }^{3}$ Department of Genetics, University of Pretoria, South Africa

${ }^{4}$ Department of Psychology, Brunel University, UK

${ }^{5}$ School of Psychology, University of St Andrews, UK

${ }^{6}$ Department of Psychology, University of Bristol, UK

\section{Corresponding author information:}

Ian D Stephen

School of Psychology,

University of Nottingham Malaysia Campus,

Jalan Broga,

43500 Semenyih,

Selangor Darul Ehsan,

Malaysia.

ian.stephen@nottingham.edu.my

Word count: 3,595

Citation details...

Stephen, I.D., Scott, I.M.L., Coetzee, V., Pound, N., Perrett, D.I. \& Penton-Voak, I.S. (2012).

Cross-cultural effects of color, but not morphological masculinity, on perceived attractiveness of men's faces, Evolution and Human Behavior, 33 (4): 260- 267. 


\section{Cross-cultural effects of color, but not morphological masculinity, on perceived attractiveness of men's faces}

\section{Summary}

Much attractiveness research has focused on face shape. The role of masculinity (which for adults is thought to be a relatively stable shape cue to developmental testosterone levels) in male facial attractiveness has been examined, with mixed results. Recent work on the perception of skin color (a more variable cue to current health status) indicates that increased skin redness, yellowness and lightness enhance apparent health. It has been suggested that stable cues such as masculinity may be less important to attractiveness judgments than short-term, more variable health cues. We examine associations between male facial attractiveness, masculinity and skin color in African and Caucasian populations. Masculinity was not found to be associated with attractiveness in either ethnic group. However, skin color was found to be an important predictor of attractiveness judgments, particularly for own-ethnicity faces. Our results suggest that more plastic health cues, such as skin color, are more important than developmental cues such as masculinity. Further, unfamiliarity with natural skin color variation in other ethnic groups may limit observers' ability to utilize these color cues.

\section{Key words}

Color, masculinity, attractiveness, health, face, perception

\section{Introduction}

A number of researchers have examined the aspects of facial appearance that affect perceived attractiveness, focusing primarily on facial shape cues such as symmetry (Perrett et al, 1999) and averageness (Rhodes et al, 1999). Sexual dimorphism in shape has also received much research interest, with studies finding that the 
femininity of women's faces is closely associated with their rated attractiveness (Rhodes, 2006; Perrett et al, 1998). However, findings regarding the attractiveness of masculine features in male faces have been more mixed.

It has been suggested that facial masculinity should enhance attractiveness in men, due to an immunohandicapping effect of testosterone ensuring that only high quality males can achieve a strongly masculine appearance during development (Hamilton \& Zuk, 1982; Lozano, 1994; see Rhodes, 2006). There is some evidence that facial masculinity is associated with levels of circulating testosterone in men (Pound et al, 2009). However, some studies have found that women prefer more feminine male faces (Perrett et al, 1998) or found no preference for masculinity (Rhodes et al, 2003). Further studies have found that women's preferences for masculinity fluctuate, for example: a) over the course of the menstrual cycle, with reduced preference for femininity in the follicular (fertile) phase, and femininity preferred in the luteal phase (Penton-Voak \& Perrett, 2000; Johnston et al, 2001; Jones et al., 2005; Penton-Voak \& Perrett, 2001; Penton-Voak et al., 1999; Scarbrough \& Johnston, 2005); b) depending on the type of relationship sought, with masculinity preferred for short-term relationships and femininity preferred for longterm relationships (Little et al, 2007); c) dependent on the attractiveness of the woman, with more attractive women preferring more masculine men (Penton-Voak et al, 2003). All of these papers posit a trade-off between gaining the "good genes" benefits of mating with masculine men and the negative personality traits (such as aggression, violence) that are associated with masculine men.

However, although it is possible that for adult males in many species aspects of anatomical masculinity may be reliable cues to health status during development, their importance may be limited in the presence of more salient cues to current health. This is particularly likely to be the case in situations where fluctuations in pathogen pressure and health status are rapid relative to host lifespan (Adamo \& Spiteri, 2005, 2009; Scott et al., 2010), and female mate choice for multiple cues is 
constrained (Kokko et al, 2003). Mathematical models developed recently indicate that for most animals, females can derive fitness benefits from paying attention to the current condition of potential mates, but may derive little or no additional benefit from attending to cues to past immune function (Adamo \& Spiteri, 2005, 2009).

Consequently, relatively stable traits that are likely not influenced substantially by short-term fluctuations in adult health (e.g. degree of anatomical masculinization; Bulygina et al, 2006) should be of less importance to females than other more condition-dependent cues to current health. Moreover, this tendency should be more pronounced in animals with long lifespans and slow reproduction, such as humans (Scott et al, 2010).

Further, recent theoretical work has suggested that the primary selective force driving the evolution of more robust features in male faces may have been intrasexual competition rather than female choice (Puts, 2010). Puts (2010) points out that the high degree of sexual dimorphism in muscle mass (similar to the dimorphism seen in gorillas; Zihlman \& MacFarland, 2000) and ability to control access to females predict that male-male contest competition would have been more important in the evolution of masculine traits than female choice. This prediction is supported by the finding that male sex-typicality on a number of traits, including beard growth (Neave \& Shields, 2008), masculine voice (Puts et al, 2006), masculine face (DeBruine et al, 2006) and brawny build (Frederick \& Haselton, 2007) increase ratings of dominance more than they do attractiveness (Puts et al, 2010). However, women are able to control mating to an extent, for example by extra-pair copulations, and consequently are predicted to favor males with healthy appearance (Puts, 2010). This adds further to the expectation that men's masculinity will contribute little to attractiveness, with women preferring cues to current health, such as color.

Recent work has shown that the distribution and homogeneity of skin color across the face contributes to perceptions of health, age and attractiveness of human faces (Fink et al, 2001, 2006; Matts et al, 2007; Stephen \& McKeegan, 2010), with 
more homogenous skin color and chromophore distribution associated with higher rated attractiveness (Fink et al, 2001; Matts et al, 2007). Overall skin color has also been shown to affect the apparent health - and likely attractiveness (Jones et al, 2004) - of human faces, with redder, yellower and lighter skin appearing healthier (Stephen et al, 2009a). The enhanced healthy appearance obtained from increased skin redness has been attributed to increased skin blood perfusion and oxygenation, which are associated with current cardiac and respiratory health (Stephen et al, 2009b) and the enhanced healthy appearance associated with increased skin yellowness has been attributed to increased levels of carotenoids, which are associated with increased resistance to reactive oxygen species (Stephen et al, 2011; Dowling \& Simmons, 2009). It is thought that color provides an indicator of current health, since the levels of pigmentation in the skin react rapidly to changes in health status. Skin carotenoid levels change within days in response to changes in dietary intake (Stahl et al, 1998) and parasitic infestation (Koutsos et al, 2003); melanin levels increase in the skin within an hour of exposure (Robins, 1991); blood oxygenation and perfusion changes rapidly in response to a number of stimuli, such as exercise and illness (Paxton et al, 1996).

Scott et al (2010) have recently shown that masculinity - a possible cue to health status during development - may not be an important predictor of attractiveness when more salient cues to current condition, such as color, are available, and that many previous findings may have been dependent largely on the experimental methods employed (e.g. using stimuli in which masculinity has been varied while other cues have been held constant). However, Scott et al (2010) used relatively wealthy participants from highly developed environments with good access to healthcare for both the stimuli and the choosers. Masculinity preferences have been shown to vary cross-culturally, with masculinity preferred more in countries with lower standards of health than in countries with high standards of health (PentonVoak et al, 2004; Scott et al, 2008; DeBruine et al, 2010), though this effect may in 
fact reflect different levels of income inequality, with more masculine features preferred in more unequal countries (Brooks et al, 2010). Potentially, masculinity may be associated with the ability to obtain and defend resources when male-male competition is high (Puts, 2010; as is the case in cultures with high resource inequality), though health standards predict masculinity preference better than do measures of violence within the United States, when income inequality is controlled (DeBruine et al, 2011).

Here, we replicate and extend Scott et al's (2010) work using two color calibrated image sets, taken from a Caucasian, UK-based population (hereon "Caucasian") and a black South African population (hereon "African"). South Africa both has lower standards of health than the UK (life expectancy is 79.01 years in the UK compared to 48.98 years in South Africa; CIA, 2009) and a greater level of wealth inequality (the UK has a Gini coefficient of 34, compared to South Africa's 65; CIA, 2009). If masculinity is an important cue of either health or access to resources, we would expect that masculinity would have a greater impact on attractiveness ratings in the African sample than in the Caucasian sample. If cues of condition are more important, we would expect that the current health indicator (skin color) would better predict attractiveness than a possible long-term health indicator (masculinity) in both Caucasian and African samples.

It has been shown that individuals are better at recognizing faces of their own ethnic group (Valentine, 1991; O'Toole et al, 1994), possibly because of greater familiarity with own-ethnicity faces (Rhodes et al, 2005). Further, participants rate own-ethnicity faces as more attractive than other-ethnicity faces (Rhodes et al, 2005). Skin color is a trait that varies widely between ethnic groups, and is markedly different between Caucasians and Africans. It may be expected that the ability to discriminate between relatively subtle skin color differences will be better when observing own-ethnicity faces than other-ethnicity faces. We predict, therefore, that skin color will predict attractiveness better in own than in other ethnicity faces. 


\section{Methods}

All research was approved by the ethics committees at the University of St Andrews and/or the University of Pretoria, as appropriate.

\section{Photography and color measurements}

We photographed 34 male Caucasian participants (ages 18-27) at the University of St Andrews, Scotland, and 41 male black African participants (ages 18-25) at the University of Pretoria, South Africa. Participants posed with neutral expressions, in a booth painted Munsell N5 gray, illuminated with 3 Verivide F20 T12/D65 daylight simulation bulbs in high frequency fixtures (Verivide, UK), to reduce the effects of flicker. The booth was located in a room with no other lighting. We placed a Munsell N5 painted board over the shoulders and included a GretagMacbeth Mini ColorChecker color chart in the frame. We color-corrected images using a leastsquares transform, from an 11-expression polynomial expansion (Hong et al. 2001) of camera RGB values for 24 ColorChecker patches to the manufacturer-specified CIELab values of the same patches. This achieved a mean color error $(\Delta E)$ of 2.44 between the 24 manufacturer stated color values and the color values obtained from the corrected images. ( $\Delta \mathrm{E}$ is the Euclidean distance between 2 color points in CIELab space, and is the standard method used for quoting color differences in CIELab color space.)

The CIELab color space is defined by L* (lightness), $a^{*}$ (redness) and b* (yellowness) color dimensions. It is modeled on the human visual system, and designed to be perceptually uniform, a change of one unit appearing to be of approximately the same magnitude regardless of its dimension (Martinkauppi, 2002). 
We used Matlab to calculate mean CIELab values across skin pixels for each face image (defining initial CIELab face color). One Caucasian face was excluded from analysis due to having skin $b^{*}$ values more than 3 standard deviations from the mean.

\section{Masculinity Measurement}

Morphometric analysis was carried out to measure the extent to which each face was typical of its sex, in a manner analogous to that used for bodies by Brown et al. (2008) and recently for faces (Scott et al. 2010). First, using criteria established by Stephan et al (2005), the $x-y$ coordinates of the 129 facial landmarks used in Scott et al (2005) were delineated for each face using Psychomorph (Tiddeman et al., 2001). Geometric morphometric techniques were then used to calculate a masculinity index for each face. Using Morphologika (O'Higgins and Jones, 1998) the Cartesian landmark coordinates were subjected to Procrustes registration - a best fit procedure that removes scale, rotational and translational differences between shapes (Gower, 1975, Rohlf and Slice, 1990, Goodall, 1991). Then, to identify dimensions of variation in facial landmark configuration, Morphologika was used to conduct Principal Components Analysis (PCA) of the Procrustes-registered landmark data. A KaiserGuttman criterion was used to select Principal Components (PCs) for inclusion in subsequent analysis; i.e. those with eigenvalues greater than the average eigenvalue were retained. This led to the retention of the first 19 PCs which together accounted for $88.5 \%$ of the variance in facial landmark configuration.

Masculinity indices were calculated within each sample to avoid confounding effects of sample differences in face shape. For each sample, step-wise discriminant analysis (SPSS 13) was used to establish which of the 19 PCs were best able to discriminate between the male and female faces. For African faces, the resulting discriminant function incorporated 5 of the PCs (Wilks' $\lambda=0.326 ; \mathrm{df}=5 ; x^{2}=81.3 .1$, $p<0.00001$ ), and yielded correct sex classifications for $97.6 \%$ of males and $100.0 \%$ 
of females. For Caucasian faces, the resulting discriminant function incorporated 12 of the PCs (Wilks' $\lambda=0.051 ; \mathrm{df}=12 ; \mathrm{X} 2=199.5, \mathrm{p}<0.00001$ ), and yielded correct sex classifications for $100 \%$ of males and females. In light of the classification accuracy, discriminant function scores were therefore used as an index of morphological masculinity, oriented such that high scores indicated a more masculine facial structure

\section{Experimentation}

Female participants were asked to rate the attractiveness of the African (15 African raters aged 18-26, 20 Caucasian raters aged 18-23) and Caucasian (15 African raters aged 18-23, 12 Caucasian raters aged 19-26) faces on a 7-point Likert-type scale from 1 (very unattractive) to 7 (very attractive). Faces were presented on a CRT monitor calibrated using a DataColor Spyder3Pro, in blocks according to ethnicity of face, and order of presentation within blocks was randomized. Caucasian raters were tested at the University of St Andrews, UK. African raters were tested at the University of Pretoria, South Africa.

\section{Statistical Methods}

Inter-rater reliability was high (Cronbach's $\alpha>0.9$ ) for raters in all four conditions (African and Caucasian raters, African and Caucasian faces). Mean attractiveness ratings were calculated for each face, and for raters of each ethnicity, so that each face had an attractiveness rating attributed by African raters and an attractiveness rating attributed by Caucasian raters.

We used linear regressions (backwards method) to identify the contribution of masculinity, $L^{*}$ (lightness), $a^{*}$ (redness) and $b^{\star}$ (yellowness) to attractiveness of faces. Each of these analyses was performed separately on attractiveness ratings by African and Caucasian raters. To check for possible curvilinear relationships between each variable and rated attractiveness, squared terms for each dependent variable 
were included in the analysis. To avoid multicollinearity caused by including both squared and linear terms in the model, masculinity and color variables were centered by subtracting the mean. All VIFs in all regression analyses were $<2$, and there were no significant correlations between the masculinity index and the CIELab $L^{*}, a^{*}$ and $b^{\star}$ variables (all $p>0.05$ ).

Since several factors have been suggested to influence individual differences in women's preferences for male masculinity - such as phase of menstrual cycle (Penton-Voak \& Perrett, 2000; Johnston et al, 2001; Jones et al., 2005; Penton-Voak \& Perrett, 2001; Penton-Voak et al., 1999; Scarbrough \& Johnston, 2005), relationship status (Little et al, 2007) and own attractiveness (Penton-Voak et al, 2003) - it may be hypothesized that different women's positive and negative preferences for masculinity may cancel each other out. If menstrual cycle effects (or other factors) leading to variation in women's preferences for masculinity are "cancelling each other out", some women should show positive relationships between their attractiveness ratings of men's faces and the morphological masculinity of those faces while others will show negative relationships. When summed, these relationships could, potentially, negate the identification of positive and negative relationships between masculinity and attractiveness in subsamples of women, masking the menstrual cycle (or other) effects. To test if this is a possibility in the current sample, Spearman's rank correlation analyses between morphological masculinity and attractiveness rating were carried out for each rater individually, following Scott et al (2010). If the "cancelling out" effect is hiding preferences for masculinity in the current sample, it is predicted that positive relationships between rated attractiveness and morphological masculinity of men's faces will be found for some women, while negative relationships will be found for other women.

\section{Results}


Table 1 summarizes the results of the linear regression analyses. Linear regressions revealed no role for morphological masculinity in predicting rated attractiveness, as neither the masculinity nor the masculinity squared term remained in the model for faces of either ethnicity, rated by raters of either ethnicity. For Caucasian faces rated by Caucasian raters, greater attractiveness was predicted by increased yellowness $\left(b^{*} ; \beta=0.658 ; p=0.032\right)$ and decreased lightness $\left(L^{*} ; \beta=-0.385 ; p=0.032\right)$ of the face. The yellowness $\left(b^{\star}\right)$ squared term remained in the model, but only as a nonsignificant trend $(\beta=-0.356 ; p=0.070)$. For African faces rated by African raters, greater attractiveness was predicted by increased yellowness $\left(b^{*} ; \beta=0.669 ; p=0.001\right)$ and decreased lightness $\left(L^{*} ; \beta=-0.475 ; p=0.011\right)$. The lightness squared term was also significant, suggesting that very light and very dark faces are not attractive $(\beta=0.669 ; p=0.001)$. Color was not found to predict rated attractiveness in Caucasian faces rated by African raters. For African faces rated by Caucasian raters, color did not significantly predict attractiveness, though the lightness $\left(L^{*}\right)$ term remained in the model $(\beta=-0.266 ; p=0.093)$.

In the individual participant analyses, only four of the 62 participants (6.5\%) showed a significant negative correlation between rated attractiveness and facial masculinity, preferring less masculine faces. The other 58 participants (>93.5\%), however, showed no such preference, suggesting that individual differences in preferences for masculinity (for example, as a result of menstrual cycle effects "cancelling each other out") cannot account for the lack of relationship between facial masculinity and rated attractiveness.

\section{Discussion}

Skin color was found to be an important predictor of facial attractiveness when participants judged faces from their own ethnic group, whereas morphological masculinity was not found to significantly predict attractiveness in own- or otherethnicity faces. This provides a cross-cultural validation, using color-calibrated 


\begin{tabular}{|c|c|c|c|}
\hline & & African raters & Caucasian raters \\
\hline African faces & Model & $\begin{array}{l}F_{3,40}=8.835 \\
p<0.001^{* * *}\end{array}$ & $\begin{array}{l}F_{1,40}=2.974 \\
p=0.093\end{array}$ \\
\hline & Masculinity & & \\
\hline & Masculinity $^{2}$ & & \\
\hline & $\mathrm{L}^{*}$ & $\beta=-0.475 ; p=0.011^{*}$ & $\begin{array}{l}\beta=-0.266 ; \\
p=0.093\end{array}$ \\
\hline & $\mathrm{L}^{\star^{2}}$ & $\begin{array}{l}\beta=0.669 \\
p=0.001^{* *}\end{array}$ & \\
\hline & $a^{*}$ & & \\
\hline & $a^{\star^{2}}$ & & \\
\hline & $\mathbf{b}^{*}$ & $\begin{array}{l}\beta=0.669 \\
p=0.001^{* *}\end{array}$ & \\
\hline & $b^{\star 2}$ & & \\
\hline Caucasian faces & Model & n.s. & $\begin{array}{l}F_{3,34}=3.732 \\
p=0.021^{*}\end{array}$ \\
\hline & Masculinity & & \\
\hline & Masculinity $^{2}$ & & \\
\hline & $\mathrm{L}^{*}$ & & $\begin{array}{l}\beta=-0.385 \\
p=0.032^{*}\end{array}$ \\
\hline & $\mathrm{L}^{\star 2}$ & & \\
\hline & $a^{*}$ & & \\
\hline & $a^{\star^{2}}$ & & \\
\hline & $\mathbf{b}^{*}$ & & $\begin{array}{l}\beta=0.658 \\
p=0.032^{\star}\end{array}$ \\
\hline & $\mathbf{b}^{\mathbf{*}^{2}}$ & & $\begin{array}{l}\beta=-0.356 \\
p=0.070\end{array}$ \\
\hline
\end{tabular}

Table 1: $\beta$ values of variables in the linear regression models, dependent variable=attractiveness rating. ${ }^{*} p<0.05,{ }^{* *} p<0.01,{ }^{* *} p<0.001$ 
images, of the findings of Scott et al (2010) that cues related to current or recent health (i.e. "state" cues) are more important predictors of facial attractiveness than structural cues to past health during development (i.e. "trait" cues; fig. 1, 2). In addition, a "cancelling out" effect of individual differences in preferences for masculinity, caused by a menstrual cycle effect (or relationship status or other factors) was not found, with more than $93.5 \%$ of participants showing no preference for high or low masculinity, It is worth noting that, since probability of conception is above $5 \%$ on 8 days of the cycle (Wilcox et al, 2000), we would expect around $28 \%$ of participants to show a preference for more masculine faces. Even assuming a high $50 \%$ rate of oral contraception among participants, 9 participants would be predicted to show a masculinity preference. In our analysis, none of the participants showed this preference. Further, mathematical modeling predicts that, even in groups containing mixed-quality females, or those judging for long-term relationships, preferences for quality would still be detectable (Hill \& Reeve, 2004). We detect preferences for color cues, but not for masculinity.

Masculinity has been found to affect attractiveness ratings in studies where only masculinity differed between faces - i.e. studies where masculinity is manipulated while other variables are held constant, even though the direction of preferences for masculinity are somewhat inconsistent (e.g. Perrett et al, 1998; Penton-Voak \& Perrett, 2000; Scott et al, 2010). However, there is only limited evidence that naturally-occurring variation in facial masculinity between individuals is an important determinant of attractiveness in the presence of other cues (e.g. Cunningham et al., 1990, Penton-Voak et al., 2001, Komori et al., 2009). It appears, therefore, that masculine facial appearance may not have evolved under selection pressure from female choice. Instead, selection pressures associated with malemale contest competition seem likely to have played a more important role (Puts, 2010). 




Figure 1: African face composite images made from the five $(A)$ Least attractive faces, rated by African raters; (B) Most attractive faces, rated by African raters; (C) Least attractive faces, rated by Caucasian raters; (D) Most attractive faces, rated by Caucasian raters; (E) Least masculine faces; (F) Most masculine faces. The more attractive composites are noticeably yellower (higher $b^{*}$ ) than the less attractive composites, especially as rated by African raters. Composites are used to illustrate typical faces of each category. Faces used in the ratings tasks and for measurements were photographs of real individuals. 




Figure 2: Caucasian face composite images made from the five $(A)$ Least attractive faces, rated by African raters; (B) Most attractive faces, rated by African

raters; (C) Least attractive faces, rated by Caucasian raters; (D) Most attractive faces, rated by Caucasian raters; $(E)$ Least masculine faces; $(F)$ Most masculine faces. The more attractive composites are noticeably yellower (higher $b^{*}$ ) than the less attractive composites. Composites are used to illustrate typical faces of each category. Faces used in the ratings tasks and for measurements were photographs of real individuals. 
Our analysis of the association between skin color and attractiveness revealed an "other-race" effect, with variation in color cues strongly predicting attractiveness in own-ethnicity faces, whilst this relationship was absent for raters viewing other-ethnicity faces. This may be attributable to a lack of familiarity with other-ethnicity faces, and therefore a lack of familiarity with the meaning of cues in other-ethnicity faces (Shepherd \& Deregowski, 1981). Moreover, the effect may be particularly strong in the present study as a consequence of the considerable difference in skin color between African and Caucasian groups which will mean that familiarity with these cues would be particularly limited (Valentine, 1991). No effect of ethnicity was found on preference for masculinity, since masculinity did not affect attractiveness perceptions in either ethnic group.

Skin redness was not found to predict attractiveness in the linear regression models. This may be due to problems of multicollinearity among the predictor variables, (skin $L^{\star}, a^{\star}$ and $b^{\star}$ values are all correlated, though tolerance and VIF values were well within acceptable levels). Multicollinearity makes it difficult to evaluate the importance of individual predictors and may be masking the effects of $a^{*}$ that have been seen when $a^{*}$ alone is manipulated (Stephen et al, 2009a,b). It is expected that the color axes will correlate with each other in human skin, since human skin color is determined by pigments - primarily melanin, carotenoids, oxygenated and deoxygenated hemoglobin - each of which impact on the $L^{*}, a^{*}$ and $b^{*}$ values of the skin. A change in the concentration of any of these pigments thus results in changes in all three color axes (Stephen et al, 2009b; 2011). It may also be the case that redness $\left(\mathrm{a}^{*}\right)$ only affects attractiveness of faces in the absence of other, more salient cues.

In conclusion, we have provided further evidence that morphological masculinity is at best a weak predictor of attractiveness ratings of male faces when variation in more salient cues to current health status, such as skin color, is present. This suggests 
that cues to current health status ("state" cues) may be more important determinants of attractiveness judgments than cues to past health status ("trait" cues), as predicted by recent models of mate choice (Adamo \& Spiteri, 2005, 2009). In addition, we have demonstrated an "other-race" effect for skin color as a predictor of attractiveness ratings, which may be attributable to an unfamiliarity with the very different skin colors of African and Caucasian individuals. Further research on skin color cues, and "other-race" effects on attractiveness would be desirable to establish whether the greater importance of color over masculinity is consistent across groups of women.

\section{Acknowledgements}

We thank Jaco Grieff for hosting work in South Africa, Lesley Ferrier for logistical support.

\section{References}

Adamo S.A., Spiteri R.J. (2005) Female choice for male immunocompetence: when is it worth it? Behavioral Ecology 16: 871-879.

Adamo S.A., Spiteri R.J. (2009) He's healthy, but will he survive the plague? Possible constraints on mate choice for disease resistance. Animal Behaviour 77: 6778.

Brooks, R, Scott, I.M., Makalov, A.A., Kasumovic, M.M. Clark, A.P. \& Penton-Voak, I.S. (2010, in press). National income inequality predicts women's preferences for masculinised faces better than health does. Proceedings of the Royal Society, B. doi:10.1098/rspb.2010.0964

Brown, W. M., Price, M. E., Kang J., Pound, N., Zhao, Y. \& Yu, H. (2008) Fluctuating asymmetry and preferences for sex-typical bodily characteristics. Proceedings of the National Academy of Sciences, 105, 12938-12943. 
Bulygina,E, Mitteroecker,P, Aiello, L (2006) Ontogeny of facial dimorphism and patterns of individual development within one human population, American Journal of Physical Anthropology, 131, 432-443.

Central Intelligence Agency, 2009 The World Factbook 2009. Washington, DC Cunningham, M. R., Barbee, A. P. \& Pike, C. L. (1990) What Do Women Want Facialmetric Assessment of Multiple Motives in the Perception of Male Facial Physical Attractiveness. Journal of Personality and Social Psychology, 59, 6172.

DeBruine, L.M., Jones, B.C., Little, A.C., Boothroyd, L.G., Perrett, D.I., Penton-Voak, I.S., Cooper, P.A., Penke, L., Feinberg, D.R. \& Tiddeman, B.P. (2006). Correlated preferences for facial masculinity and ideal or actual partner's masculinity. Proceedings of the Royal Society of London B, 273: 1355-1360.

DeBruine, L.M., Jones, B.C., Crawford, J.R., Welling, L.L.M. \& Little, A.C. (2010).

The health of a nation predicts their mate preferences: Cross-cultural variation in women's preferences for masculinized male faces. Proceedings of the Royal Society of London B, 277, 2405-2410.

DeBruine, L.M., Jones, B.C., Little, A.C., Crawford, J.R. \& Welling, L.L.M. (2011). Further evidence for regional variation in women's masculinity preferences. Proceedings of the Royal Society of London B, 728, 813-814.

Dowling, D.K. \& Simmons, L.W. (2009) Reactive oxygen species as universal constraints in life-history evolution. Proc. R. Soc. Lond. B, 276, 1737-1745. Dryden IL, Mardia KV (1998) Statistical Shape Analysis (John Wiley and Sons, London).

Fink, B., Grammer, K., \& Thornhill, R. (2001). Human (Homo sapiens) facial attractiveness in relation to skin texture and color. Journal of Comparative Psychology, 115(1), 92-99. 
Fink, B., Grammer, K., \& Matts, P. J. (2006). Visible skin color distribution plays a role in the perception of age, attractiveness and health in female faces. Evolution and Human Behavior, 27(6), 433-442.

Goodall, C. (1991) Procrustes Methods in the Statistical Analysis of Shape. Journal of the Royal Statistical Society. Series B (Methodological), 53, 285-339.

Gower, J. (1975) Generalized procrustes analysis. Psychometrika, 40, 33-51.

Hamilton, W. D., \& Zuk, M. (1982). Heritable true fitness and bright birds: a role for parasites? Science, 218, 384-387.

Hill, S.E. \& Reeve, K. (2004) Mating games: the evolution of human mating transactions. Behavioural Ecology, 15, 748-756.

Hong, G., Luo, M. R., \& Rhodes, P. A. (2001). A study of digital camera colorimetric characterization based on polynomial modeling. Color Research \& Application, 26(1), 76-84.

Johnston, V. S., Hagel, R., Franklin, M., Fink, B., \& Grammer, K. (2001). Male facial attractiveness: Evidence of hormone-mediated adaptive design. Evolution and Human Behavior, 22, 251-267.

Jones, B. C., Little, A. C., Burt, D. M., \& Perrett, D. I. (2004). When facial attractiveness is only skin deep. Perception, 33, 569-576.

Kokko H, Brooks R, Jennions M, Morley J, 2003. The evolution of mate choice and mating biases. Proc R Soc Lond B 270:653-664.

Komori, M., Kawamura, S. \& Ishihara, S. (2009) Effect of averageness and sexual dimorphism on the judgment of facial attractiveness. Vision Research, 49, 862-869.

Koutsos, E. A., Calvert, C. C. and Klasing, K. C. (2003). The effect of an acute phase response on tissue carotenoid levels of growing chickens (Gallus gallus domesticus). Comp. Biochem. Physiol. 135A, 635-646. 
Little AC, Cohen D, Jones BC, \& Belsky J. (2007). Human preferences for facial masculinity change with relationship type and environmental harshness. Behavioural Ecology and Sociobiology 61, 967-973.

Lozano, G. A. (1994). Carotenoids, Parasites, and Sexual Selection. Oikos, 70(2), 309-311.

Martinkauppi B (2002) Face color under varying illumination-analysis and applications. Oulu: Oulu University Press.

Matts, P. J., Fink, B., Grammer, K., \& Burquest, M. (2007). Color homogeneity and visual perception of age, health and attractiveness of female facial skin. J Am Acad Dermatol, 57(6), 977-984.

Neave, N., \& Shields, K. (2008). The effects of facial hair manipulation on female perceptions of attractiveness, masculinity and dominance in male faces. Personality and Individual Differences, 45, 373-377.

O'Higgins, P. \& Jones, N. (1998) Facial growth in Cercocebus torquatus: an application of three-dimensional geometric morphometric techniques to the study of morphological variation. Journal of Anatomy, 193, 251-272.

O'Toole, A.J., Deffenbacher, K.A., Valentin, D., Abdi, H. (1994) Structural aspects of face recognition and the other-race effect. Memory and Cognition, 22, 208224.

Paxton, L.A., Redd, S.C., Steketee, R.W., Otieno, J.O., Nahlen, B. (1996) An evaluation of clinical indicators for severe paediatric illness. Bulletin of the World Health Organisation, 74, 613-618.

Penton-Voak, I. S., Perrett, D. I., Castles, D. L., Kobayashi, T., Burt, D. M., Murray, L. K., et al. (1999). Menstrual cycle alters face preference. Nature, 399, 741742.

Penton-Voak, I. S., \& Perrett, D. I. (2000). Female preference for male faces changes cyclically: Further evidence. Evolution and Human Behavior, 21(1), 39-48. 
Penton-Voak, I. S., Jones, B. C., Little, A. C., Baker, S., Tiddeman, B., Burt, D. M. \& Perrett, D. I. (2001) Symmetry, sexual dimorphism in facial proportions and male facial attractiveness. Proceedings of the Royal Society of London Series B-Biological Sciences, 268, 1617-1623.

Penton-Voak, I. S., \& Perrett, D. I. (2001). Male facial attractiveness: Perceived personality and shifting female preferences for male traits across the menstrual cycle. Advances in the Study of Behavior, 30, 219-259.

Penton-Voak, I. S., Little, A. C., Jones, B. C., Burt, D. M., Tiddeman, B. P., \& Perrett, D. I. (2003). Female condition influences preferences for sexual dimorphism in faces of male humans (Homo sapiens). Journal of Comparative Psychology, 117(3), 264-271.

Penton-Voak, I. S., Jacobson, A., \& Trivers, R. (2004). Populational differences in attractiveness judgments of male and female faces: Comparing British and Jamaican samples. Evolution And Human Behavior, 25(6), 355-370.

Perrett, D. I., Burt, D. M., Penton-Voak, I. S., Lee, K. J., Rowland, D. A., \& Edwards, R. (1999). Symmetry and Human Facial Attractiveness. Evolution and Human Behavior, 20(5), 295-307.

Perrett, D. I., Lee, K. J., Penton-Voak, I., Rowland, D., Yoshikawa, S., Burt, D. M., et al. (1998). Effects of sexual dimorphism on facial attractiveness. Nature, 394(6696), 884-887.

Pound, N., Penton-Voak, I.S., \& Surridge, A. K. (2009). Testosterone responses to competition in men are related to facial masculinity. Proceedings of the Royal Society: Biological Sciences, 276, 153-159

Puts, D.A. (2010) Beauty and the beast: mechanisms of sexual selection in humans. Evolution and Human Behavior, 31, 157-175.

Rhodes, G., Sumich, A., \& Byatt, G. (1999). Are Average Facial Configurations Attractive Only Because of Their Symmetry? Psychological Science, 10(1), $52-58$. 
Rhodes, G., Chan, J., Zebrowitz, L. A., \& Simmons, L. W. (2003). Does sexual dimorphism in human faces signal health? Proceedings of the Royal Society B: Biological Sciences, 270(0), S93-S95.

Rhodes, G., Lee, K., Palermo, R., Weiss, M, Yoshikawa, S., Clissa, P., Williams, T., Peters, M., Winkler, C., Jeffery, L. (2005) Attractiveness of own-race, otherrace and mixed-race faces. Perception, 34, 319-340.

Rhodes, G. (2006) The evolutionary psychology of facial beauty. Annual Review of Psychology, 57, 199-226.

Robins, A. (1991) Biological Perspectives on Human Pigmentation. Cambridge University Press, Cambridge.

Rohlf, F. J. \& Slice, D. (1990) Extensions of the Procrustes Method for the Optimal Superimposition of Landmarks. Syst Biol, 39, 40-59.

Scarbrough, P. S., \& Johnston, V. S. (2005). Individual differences in women's facial preferences as a function of digit ratio and mental rotation ability. Evolution and Human Behavior, 26(6), 509-526.

Scott, I.M.L., Swami, V., Josephson, S. C., \& Penton-Voak, I. S. (2008). Contextdependent preferences for facial dimorphism in a rural Malaysian population. Evolution and Human Behavior, 29, 289-296.

Scott, I.M.L., Pound, N., Stephen, I.D., Penton-Voak, I.S. (2010) Does masculinity matter? The contribution of sex-typical appearance to male attractiveness in humans. PLoS one

Shepherd, J.W., \& Deregowski, J.B. (1981) Races and faces: a comparison of the responses of Africans and Europeans to faces of the same and different races. British Journal of Psychology, 20, 125-133.

Stahl, W., Heinrich, U., Jungmann, H., von Laar, J., \& Schietzel, M., et al. (1998). Increased dermal carotenoid levels assessed by noninvasive reflection spectrophotometry correlate with serum levels in women ingesting Betatene. Journal of Nutrition, 128, 903-907. 
Stephan, C. N., Penton-Voak, I. S., Perrett, D. I., Tiddeman, B. P., Clement, J. G. \& Henneberg, M. (2005) Two-Dimensional Computer Generated Average Human Face Morphology and Facial Approximation. IN Marks, M. \& Clement, J. (Eds.) Computer Graphic Facial Reconstruction. Elsevier Science.

Stephen, I.D., McKeegan, A.M. (2010) Lip color affects perceived sex typicality and attractiveness of human faces. Perception.

Stephen, I.D., Law Smith, M.J., Stirrat, M.R., Perrett, D.I. (2009a) Facial skin coloration affects perceived health of human faces. Int. J. Primatol., 30, 845857.

Stephen, I. D., Coetzee, V., Law Smith, M. J., Perrett, D. I. (2009b) Skin blood perfusion and oxygenation color affect perceived human health. PLOS ONE, 4, e5083.

Stephen, I.D., Coetzee, V., Perrett, D.I. (2011) Carotenoid and melanin pigment coloration affect perceived human health. Evolution \& Human Behavior.

Tiddeman, B., Burt, D. M. \& Perrett, D. I. (2001) Prototyping and Transforming Facial Textures for Perception Research. IEEE Computer Graphics and Applications, 21, 42-50.

Valentine, T. (1991) A unified account of the effects of distinctiveness, inversion and and race in face recognition. Quarterly Journal of Experimental Psychology, 43A, 161-204.

Wilcox, A.J., Dunson, D., Baird, D.D. (2000) The timing of the "fertile window" in the menstrual cycle: dey specific estimates from a prospective study. British Medical Journal, 321, 1259.

Zihlman, A. L., \& MacFarland, R. K. (2000). Body mass in lowland gorillas: A quantitative analysis. American Journal of Physical Anthropology, 113, 61-78. 